\title{
Generalized temporal sampling with active illumination in optical microscopy
}

\author{
Christian Jaques ${ }^{1,2}$ and Michael Liebling ${ }^{1,3}$ \\ ${ }^{1}$ Idiap Research Institute, Switzerland \\ 2 École Polytechnique Fédérale de Lausanne, Switzerland \\ ${ }^{3}$ Department of Electrical and Computer Engineering, University of California, Santa Barbara
}

\begin{abstract}
Generalized sampling is a flexible framework for signal acquisition, which relaxes the need for ideal pre-filters. Nevertheless, implementation remains challenging for dynamic imaging applications because it requires simultaneously measuring multiple overlapping inner-products and because only positive signals (intensities) can be measured by cameras. We present a method to collect videos of monochromatic objects by projecting the incoming signal at each pixel in a temporal B-spline space of degree 0,1 , or 2 by using a conventional RGB camera and a modulated three-color light source for illumination. Specifically, we solve the basis function overlap problem by multiplexing the acquisition in different color ranges and use B-spline pieces (which are positive) as projection kernels of a biorthogonal projection-expansion bases pair. The steps to recover signal samples include spectral unmixing and inverse filtering. Reconstructions we obtained from simulated and experimentally-acquired microscopy data demonstrate the feasibility of our approach.
\end{abstract}

Index Terms - Generalized sampling, B-splines, wavelets, computational imaging, microscopy, spectral unmixing.

\section{INTRODUCTION}

Observing phenomena in live biological samples in microscopy requires sufficient time-resolution. ${ }^{1}$ Besides the development of faster and more sensitive cameras and clever pixel rebinning methods, ${ }^{2}$ various sensing and computational approaches to increase the temporal resolution of microscopes have been proposed. Some rely on multiple observations of a signal ${ }^{3-6}$ or make clever use of the signal structure itself, e.g. its sparsity in a known basis ${ }^{7-9}$ or its repeatable nature. ${ }^{10,11}$ The ability to modulate the illumination rapidly in a controlled and cost-effective way (in particular, with LED-based illuminators ${ }^{12}$ ) also opens the way for promising methods. For instance, short light pulses (stroboscopy) have been used to reduce motion blur ${ }^{13}$ or the fluttered shutter principle $^{14,15}$ uses a pseudo-random temporal illumination sequence to computationally improve the temporal resolution.

Despite the above developments, many imaging systems still rely on direct image acquisition, which is vulnerable to aliasing if the imaged signal contains frequencies higher than the Nyquist frequency, since perfect low-pass filters cannot be implemented in practice. Generalized sampling ${ }^{16}$ offers a framework to implement sampling operations that relaxes the need for ideal filters. Applications that build upon this framework have been proposed in optics ${ }^{17}$ or to sample signals with a finite rate of innovation on multiple channels. ${ }^{18}$

Due to the shift-invariant nature of generalized sampling, the implementation of pre-filters in time can be problematic because of the temporal overlap of the inner product kernels. Indeed, if the pre-filter used is longer than a unit of time (sampling interval), several inner-products must be carried out simultaneously, which requires a multiplexed acquisition approach. Moreover, in standard (incoherent light) optical imaging applications, only the intensity of the light, which is always positive can be measured. While modulation is possible by illuminating the sample with a variable intensity over time, this illumination suffers from the same positivity limitations (only positive illumination functions can be considered). In this paper, we present an approach to overcome the above positivity and multiplexing limitations to carry out generalized sampling in the context of optical microscopy

Correspondance: \{first name\}.\{last name\}@idiap.ch 
imaging. We propose to use active multicolor illumination and a color camera for collection, allowing spectral multiplexing and ideal prefiltering of the signal. Specifically, we modulate the illumination signal over time with independent signals in different color channels of the illumination lamps, which produces modulated signals whose integration by a camera shutter can be converted to the inner product between the signal and the prefilter kernel. Following recovery of these coefficients and reconstruction of the signal in the projection basis' dual basis, we obtain samples of the incoming signal, projected on the space spanned by a shift-invariant B-spline basis.

This paper is organized as follows. In Section 2, we provide a formal description of our problem. In Section 3, we derive our method. In Section 4, we illustrate our approach on both synthetic signals and from data collected on a transmission wide-field microscope. Finally, we conclude in Section 5.

\section{PROBLEM STATEMENT}

We consider a continuous-time signal $f(t)$, of which we want to estimate the least-squares approximation $\tilde{f}(t)$ in the shift-invariant space spanned by B-splines of degree $n, V=\left\{\beta^{n}(\cdot-k), k \in \mathbb{Z}\right\}$, which can be obtained via a biorthogonal projection: ${ }^{16}$

$$
\tilde{f}(t)=\sum_{k \in \mathbb{Z}} \underbrace{\left\langle f, \stackrel{\circ}{\beta}^{n}(\cdot-k)\right\rangle}_{c[k]} \beta^{n}(t-k),
$$

with

$$
\langle f, g\rangle=\int_{-\infty}^{\infty} f(t) g(t) \mathrm{d} t
$$

and where $\stackrel{\circ}{\beta}^{n}(t)$ is the dual B-spline, ${ }^{19}$ a function which, in addition to satisfying,

$$
\left\langle\beta^{n}(\cdot-\ell), \stackrel{\circ}{\beta}^{n}(\cdot-k)\right\rangle=\delta[\ell-k],
$$

also spans the subspace $V$ (and is therefore unique).

A practical system should therefore provide the measurements $c_{k}$, which correspond to weighted integrals of the input signals. There are two issues when implementing this. First, the $\AA^{n}$ have infinite support and hence overlap in time (except for degree $n=0$ ), which requires some way of splitting the input signal to perform the inner products in parallel, and, second, the signals in this setting are light intensities, that have to be positive.

To implement the projection settings, we consider a conventional imaging system with a camera that has $C$ color channels (e.g. an RGB pixel of a color camera corresponds to $C=3$ ). We further assume that the system has $L$ light sources that uniformly illuminate the scene, each with a different, yet possibly overlapping, color spectrum. The intensity of each light source can be controlled as a function of time and independently. These functions can only be positive (as light intensities are necesarily positive) and their duration should be less than the integration time of each of the camera's frames. We model the imaging system by taking into account the crosstalk that arises from the use of broadband light sources and wide camera (RGB) filters. For a single pixel at time $k$, we simultaneously measure the intensities $y_{c}[k]$ in channels $c=1, \ldots, C$ :

$$
\left(\begin{array}{c}
y_{1}[k] \\
\vdots \\
y_{C}[k]
\end{array}\right)=\left(\begin{array}{cccc}
\gamma_{1,1} & \cdots & \gamma_{1, L} & D_{1} \\
\vdots & \ddots & \vdots & \vdots \\
\gamma_{C, 1} & \cdots & \gamma_{C, L} & D_{C}
\end{array}\right)\left(\begin{array}{c}
a_{1}[k] \\
\vdots \\
a_{L}[k] \\
1
\end{array}\right)
$$

or in condensed form:

$$
\boldsymbol{y}=\boldsymbol{\Gamma} \boldsymbol{a},
$$

where the matrix $\boldsymbol{\Gamma}$ contains the crosstalk mixing terms (including an affine offset) and the coefficients $a_{\ell}[k]$ are the inner products:

$$
a_{\ell}[k]=\left\langle f, s_{\ell}(\bullet-k)\right\rangle .
$$

where $s_{\ell}(t)$ are the positive illumination functions, whose support covers the sensor exposure interval.

With this formulation, the problem of recovering the samples $c[k]$ in Equation (1) from measurements $y_{c}[k]$, can be broken down into the following sub-problems: 

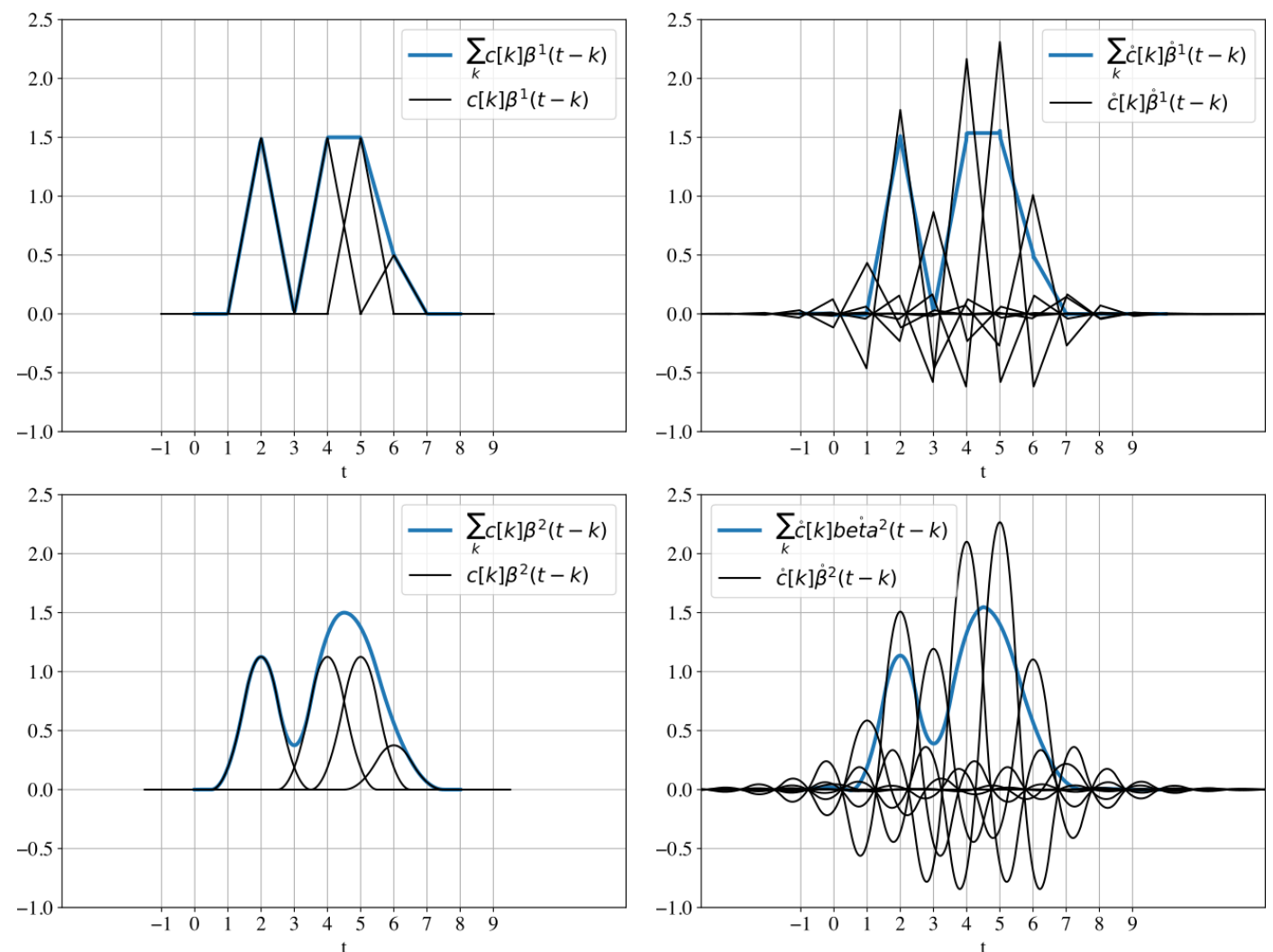

Figure 1. Equivalent basic and dual B-spline representations of the same signal for degrees $n=1$ (top row) and $n=$ 2 (bottom row). Notice that both represented signals (in blue in all plots) are equivalent, i.e. $\sum_{k} c[k] \beta^{n}(t-k)=$ $\sum_{k} \stackrel{\circ}{c}[k] \beta^{n}(t-k)$, while the basic B-spline representation uses functions of finite support.

1. find suitable illumination functions $s_{\ell}$ (in particular, positive and with a finite support) such that the sequence $c[k]$ can be derived from the multi-channel sequence $a_{\ell}[k]$.

2. determine the coefficients $c[k]$ from the coefficients $a_{\ell}[k]$ (via inverse filtering)

3. determine the coefficients $a_{\ell}[k]$ from the $y_{c}[k]$ (via spectral unmixing).

We detail these steps in the section below.

\section{METHODS}

\subsection{Multi-color B-spline segments as prefilter kernels}

Our goal is is to compute the inner products in Eq. (1) via active illumination by choosing illumination functions $s_{\ell}(t-k)$ appropriately. The challenge of this task is that we cannot use $s_{\ell}(t-k)=\dot{\beta}^{n}(t-k)$ directly (which would be the natural choice) because the dual of a B-spline is not positive for all $t$ (except when $n=0$ ) and $s_{\ell}(t)$ being a light intensity, it must be positive. We work around this problem by using an equivalent representation of the projection described in Equation (1) and illustrated in Figure 1, by switching the role of the dual bases ${ }^{19}$ namely:

$$
\tilde{f}(t)=\sum_{k \in \mathbb{Z}}\left\langle f, \stackrel{\circ}{\beta}^{n}(\cdot-k)\right\rangle \beta^{n}(t-k)=\sum_{k \in \mathbb{Z}}\left\langle f, \beta^{n}(\cdot-k)\right\rangle \stackrel{\circ}{ }^{n}(t-k) .
$$

Specifically, the coefficients in the dual B-spline basis are given by:

$$
\stackrel{\circ}{c}[k]=\left\langle\beta^{n}(\cdot-k), f\right\rangle=\int_{-\frac{n+1}{2}}^{\frac{n+1}{2}} \beta^{n}(t-k) f(t) \mathrm{d} t,
$$



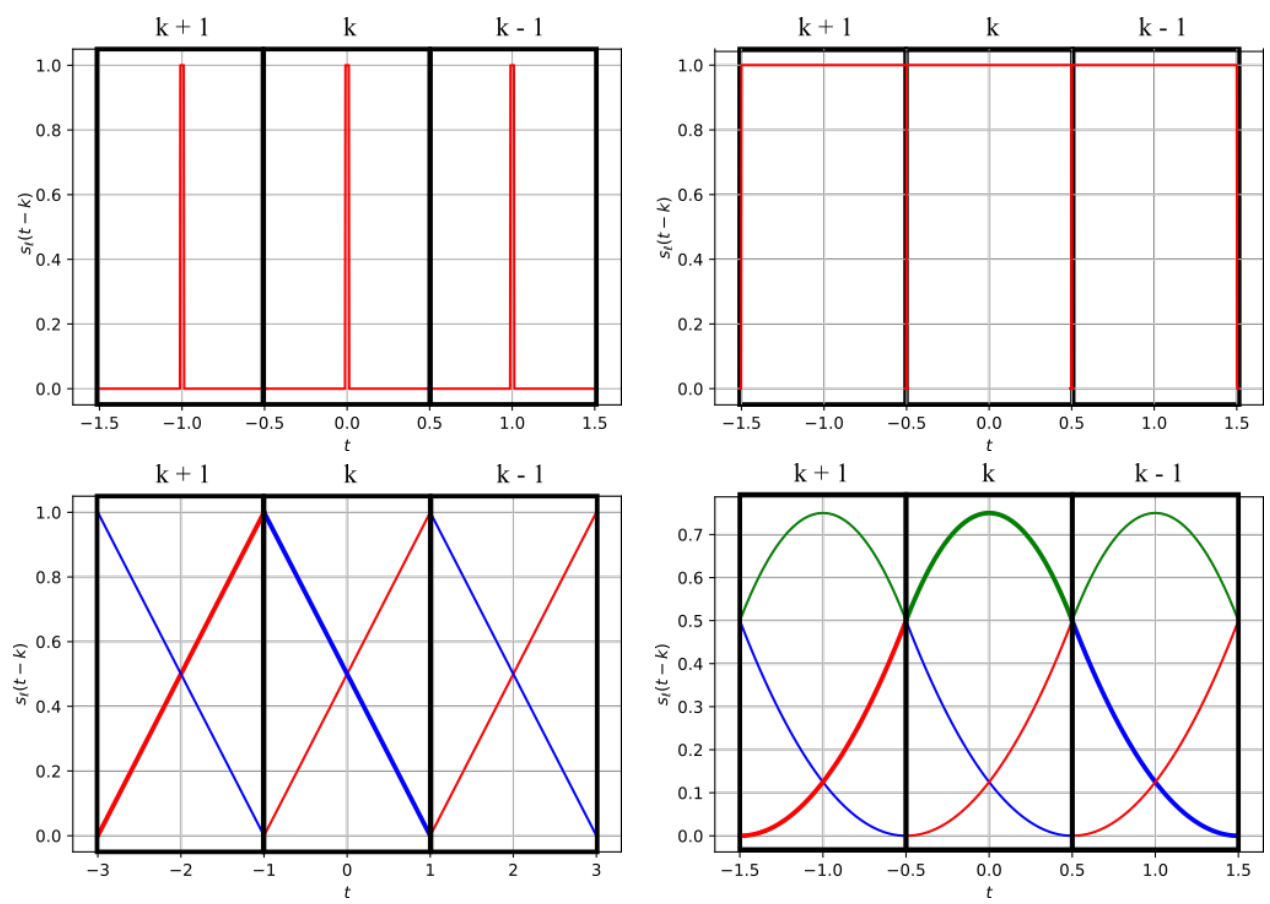

Figure 2. Illumination functions $s_{\ell}(t-k)$ over three frames for stroboscopic imaging (top left), $n=0$ (top right), $n=1$ (bottom left), and $n=2$ (bottom right). The color of the line corresponds to the color of the illumination light. For $n=1$ and $n=2$ (bottom plots), multiple frames are involved to compute a single coefficient $\stackrel{\AA}{c}[k]$ from the coefficients $a_{\ell}$.

where we note that all involved functions are positive and, since the B-spline have a finite support, the innerproduct can be computed over a finite interval. Nevertheless, since the support of B-splines of degree $n$ is $n+1$, we have $n+1$ shifted B-splines that overlap in the signal representation at any given time (see Figure 1) making the sequential computation of the $\stackrel{\ominus}{c}[k]$ problematic. In order to still acquire several inner products simultaneously, we spectrally multiplex the measurements according to Equation (4), by splitting each B-spline into $n+1$ regions, which gives the following illumination functions $s_{\ell}(t)$, for $n$ illumination sources $(\ell=1, \ldots, n)$; for $n=0$, we have

$$
s_{1}(t)=\beta^{0}(t)
$$

for $n=1$, we have:

$$
\begin{aligned}
& s_{1}(t)= \begin{cases}\beta^{1}(t)=1-t, & 0<t<1 \\
0, & \text { otherwise }\end{cases} \\
& s_{2}(t)= \begin{cases}\beta^{1}(t-1)=t, & 0<t<1 \\
0, & \text { otherwise; }\end{cases}
\end{aligned}
$$

and for $n=2$, we have

$$
\begin{aligned}
& s_{1}(t)= \begin{cases}\beta^{2}(t+1)=\frac{1}{2}\left(\frac{1}{2}-t\right)^{2} & -1 / 2<t<1 / 2 \\
0, & \text { otherwise, }\end{cases} \\
& s_{2}(t)= \begin{cases}\beta^{2}(t)=\frac{3}{4}-t^{2}, & -1 / 2<t<1 / 2 \\
0, & \text { otherwise, }\end{cases} \\
& s_{3}(t)= \begin{cases}\beta^{2}(t-1)=\frac{1}{2}\left(\frac{1}{2}+t\right)^{2} & -1 / 2<t<1 / 2 \\
0, & \text { otherwise, }\end{cases}
\end{aligned}
$$


where

$$
\beta^{2}(t)= \begin{cases}\frac{1}{2}\left(\frac{3}{2}-|t|\right)^{2}, & \frac{1}{2}<|t|<\frac{3}{2} \\ \frac{3}{4}-t^{2}, & |t| \leq \frac{1}{2} \\ 0, & \text { otherwise. }\end{cases}
$$

Figure 2 shows the illumination functions over three consecutive acquired frames for $n=0 n=1$, and $n=2$. The color of the line corresponds to the color of the illumination. Notice that in order to reconstruct $\stackrel{\circ}{c}[k]$ we have to combine measurements over multiple frames (see the bold lines in Fig. 2 for $n=1$ and $n=2$ ).

The red illumination is light $1\left(a_{1}\right)$, the blue illumination is light $2\left(a_{2}\right)$ and the green illumination is light 3 $\left(a_{3}\right)$. For degrees $n=0,1$ and 2 the coefficients $\stackrel{\circ}{c}[k]$ can be recovered from $a_{\ell}[k]$ as described below (assuming mirror boundary conditions).

Degree $n=0$ :

$$
\stackrel{\circ}{c}[k]=a_{1}[k]
$$

Degree $n=1$ :

$$
\stackrel{c}{c}[k]= \begin{cases}a_{1}[k]+a_{2}[k+1], & 0 \leq k<K-1 \\ 2 a_{1}[K-1], & k=K-1\end{cases}
$$

Degree $n=2$ :

$$
\stackrel{\circ}{ }[k]= \begin{cases}a_{1}[k-1]+a_{3}[k]+a_{2}[k+1], & 1 \leq k<K-2 \\ a_{2}[0]+a_{3}[0]+a_{2}[1], & k=0 \\ a_{1}[K-1]+a_{1}[K-1]+a_{1}[K-2], & k=K-1\end{cases}
$$

\subsection{Spectral unmixing}

The spectral unmixing procedure is similar to the setting described in Jaques et al. ${ }^{20}$ Specifically, we recover the vector $\boldsymbol{a}$ by solving the minimization problem:

$$
\boldsymbol{a}^{\star}=\min _{\boldsymbol{a}}\|\boldsymbol{Y}-\boldsymbol{D}-\boldsymbol{\Gamma} \boldsymbol{a}\|_{2}^{2} .
$$

\subsection{Converting to samples}

We obtain the basic B-spline representation through filtering of the coefficients in the dual-spline representation: ${ }^{19}$

$$
c[k]=\left(b^{2 n+1}\right)^{-1} * \stackrel{\circ}{c}[k] .
$$

We can further obtain samples by carrying out interpolation

$$
\tilde{f}[k]=\left.\tilde{f}(t)\right|_{t=k}=c * b_{1}^{n}[k],
$$

where

$$
b_{1}^{n}[k]=\left.\beta^{n}(t)\right|_{t=k} .
$$




$$
\beta^{0}(t-5)+\beta^{1}(t-10)+\beta^{2}(t-15)+\beta^{3}(t-20)
$$
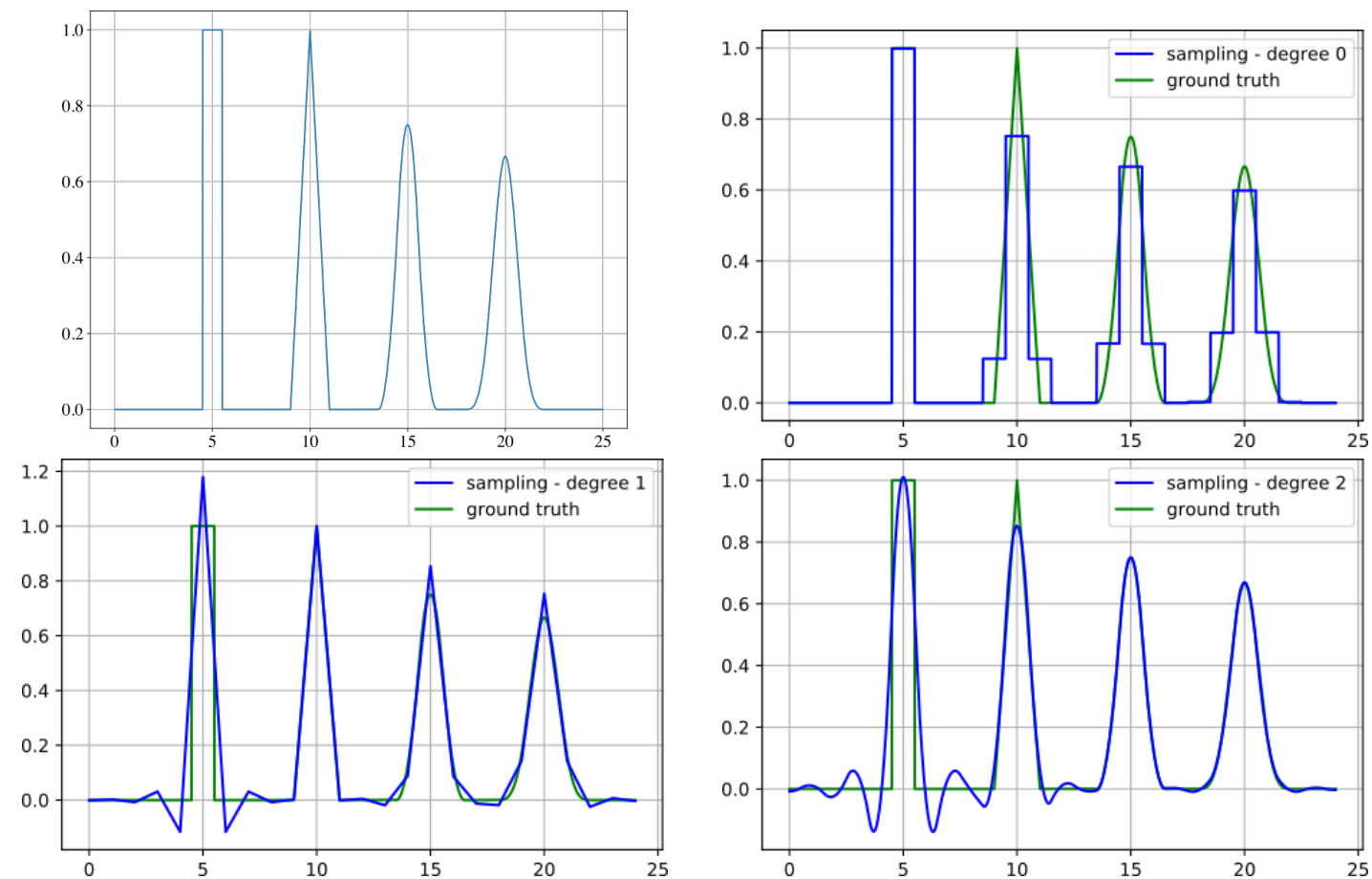

Figure 3. Shifted B-splines of degrees 0, 1 and 2. We observe that each B-spline is perfectly reconstructed when the sampling is done with B-splines of the same degree.

\section{EXPERIMENTS}

\subsection{Reconstructions from synthetic data}

We conducted an experiment to illustrate the impact of the chosen sampling basis. We generated a 1D signal containing shifted B-splines of various degrees, shown on Figure 3. We then simulated active sampling using B-splines of degrees 0 to 2 as pre-filters. Figure 3 shows the reconstructions obtained by sampling the signal on the top left of the figure. We observe that the B-splines in the sampled signal are perfectly reconstructed when the pre-filter is of the same degree than the B-spline. We also see overshooting and ringing after sharp transitions, for degrees $n \geq 1$.

We set out to investigate the effect of our method in the presence of high frequencies, that we compared to a stroboscopic imaging system. We generated a 1D temporal chirp signal and simulated sampling with B-splines of degree 2 as well as stroboscopic imaging. The signal and sampling simulations are shown on Fig. 4, where we notice that stroboscopic imaging is strongly subject to aliasing, while generalized sampling by projection on B-spline bases gracefully handles higher frequencies.

\subsection{Microscopy}

\subsubsection{Hardware and parameters setup}

We implemented the illumination with commonly available and cost-effective hardware. We assembled a light source using a 6-LED chip (SLS Lighting RGBWA+UV, Aliexpress, China). For the experiment, we drove the red $(\lambda \approx 620 \mathrm{~nm})$, green $(\lambda \approx 525 \mathrm{~nm})$ and blue $(\lambda \approx 465 \mathrm{~nm})$ LEDs via a micro-controller (Arduino Uno, Arduino, Italy), which we programmed to generate the illumination time-pattern shown on Figure 2, individually controlling each color. For the LED-camera synchronization, the micro-controller monitored the flash trigger output of the camera. Whenever the trigger signal transitions from low to high state, the micro-controller starts the time-sequence of the LEDs for the frame about to be recorded. The LEDs were directly powered by the controller's outputs, without additional power amplification of the signal. 

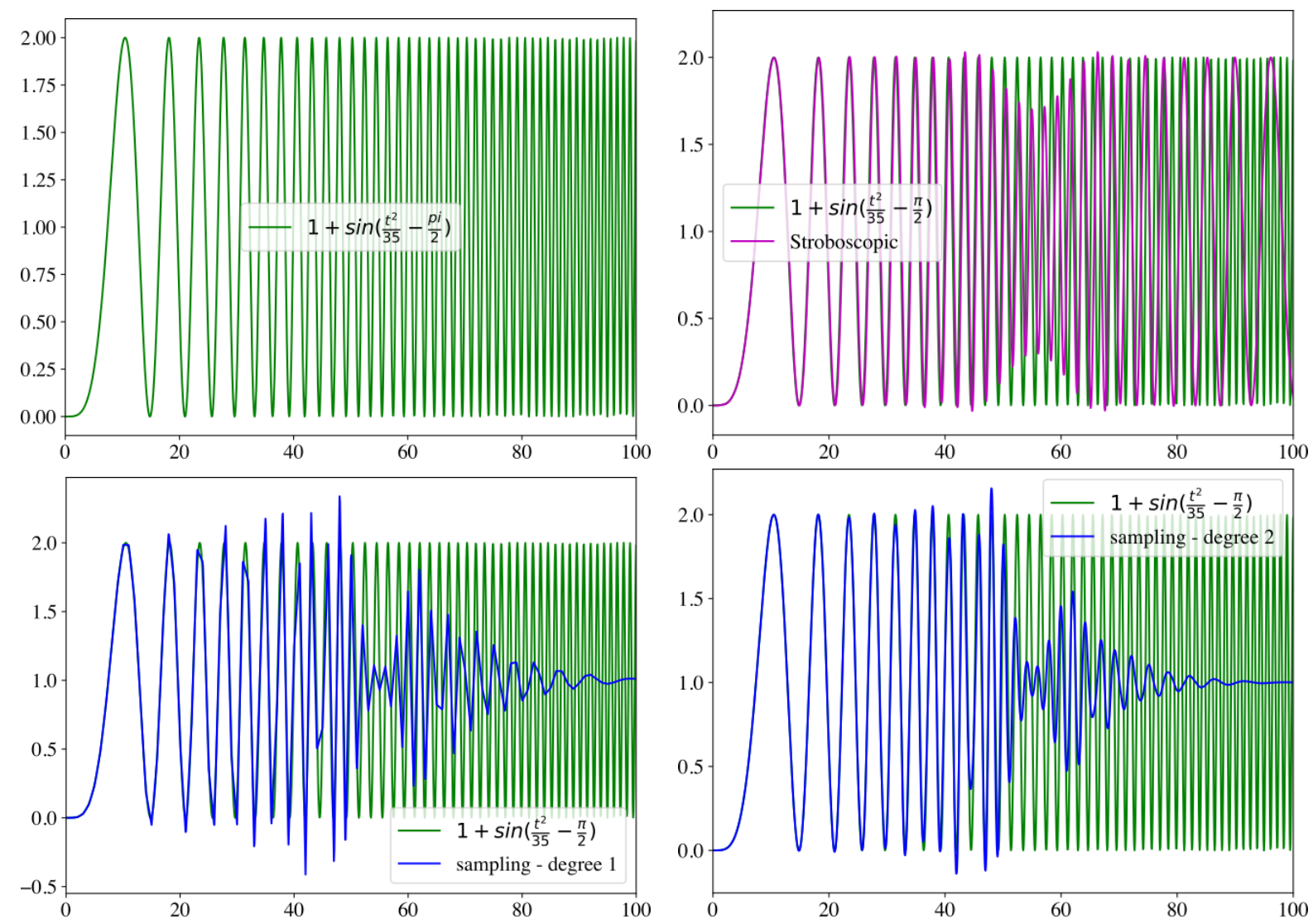

Figure 4. Sampling simulations of the signal $f(t)=1+\sin \left(\frac{t^{2}}{35}+\frac{\pi}{2}\right)$ (top left). We simulated stroboscopic imaging (top right) and generalized sampling with B-splines of degree 1 (bottom left) and 2 (bottom right). Notice that the B-spline sampling reconstructions for high frequencies $(t \geq 80)$ goes to the average of the signal, while the stroboscopic imaging shows aliasing.

We used a CMOS color camera (Thorlabs DCC3240C, Thorlabs, Germany) with $1280 \times 1024$ pixels and a standard RGGB-Bayer filter pattern. We attached the camera to the camera port of a custom-built wide-field transmission microscope (based on OpenSPIM ${ }^{21}$ ) consisting of a $20 \times$ Olympus water dipping lens (Olympus Plan Fluorite UMPLFLN 20xW) combined with a $180 \mathrm{~mm}$ tube lens (Olympus U-TLU-1-2) ended by a $0.5 \times$ lens (Olympus U-TV0.5XC-3). We set the exposure of our camera to $E=120$ milliseconds.

\subsubsection{Temporal generalized sampling for microscopy}

We mounted a transparent sheet with black stripes printed on it under our microscope in transmission mode, and set it lateral motion using a motorized positioning stage (USB $4 D$-STAGE, Picard Industries, USA). Thus, when the samples moved and a pixel was suddenly covered (or uncovered) by a black stripe the generated signal was equivalent to a step function in time, with intensity going from from 1 (white) to 0 (black). Figure $5(\mathrm{~g}$ ) shows reconstructions based on projections in B-spline spaces of degrees 0,1 , and 2, obtained in separate runs of the experiment (each with its corresponding illumination pattern), as well as images obtained via stroboscopic imaging. All curves correspond to the same location on the sample and were temporally shifted to match one another for comparison.

Although the sharpest images are obtained via a strobed illumination, this approach would be subject to aliasing at higher speeds, as illustrated in the simulations (Fig. 4). Here, since the grid moves by 200 pixels between each frame, while the distance between two white stripes is of 440 pixels leading to $f_{\text {Nyquist }}=\frac{2 \cdot 200}{440} \leq 1$. Reconstructions show higher oscillations as the degree of the projection increases. 

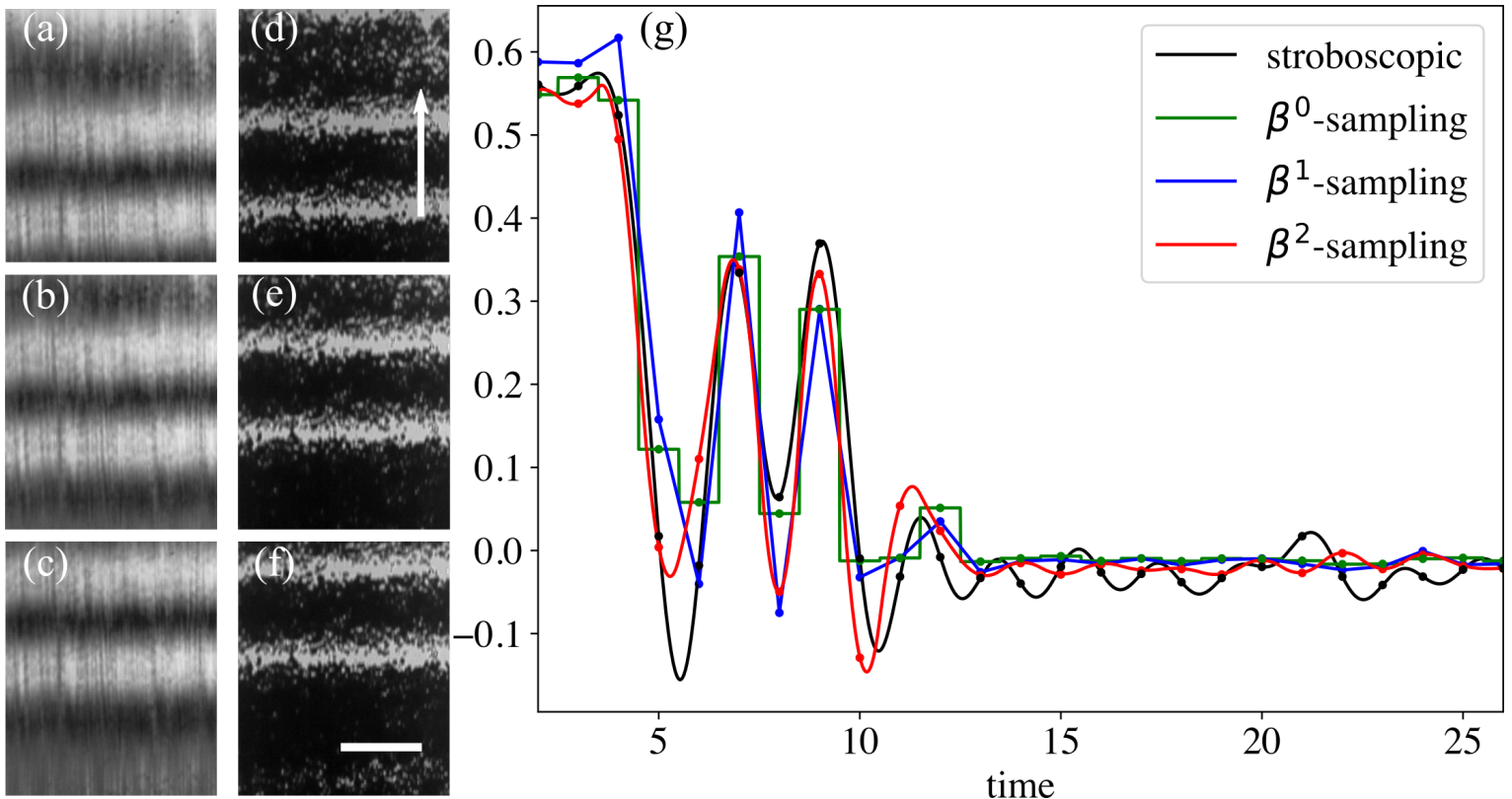

Figure 5. (a),(b),(c) Image reconstructions using B-spline pre-filters of degree $n=1$ for three consecutive time points. (d),(e),(f) Stroboscopic imaging of the same sample for the similar three time-points. Notice that the stroboscopic acquired images are sharper than the ones from B-spline sampling, but they remain subject to temporal aliasing. The arrow in (d) shows the direction of motion of the sample. (g) Temporally interpolated signals for various degrees at the same spatial location. Note the oscillations with the stroboscopic imaging and B-spline sampling of degree 2. Scalebar: $200 \mu \mathrm{m}$.

\section{CONCLUSION}

We presented a method to perform temporal generalized sampling in optical microscopy. Our approach allows for the use of implementable pre-filters with finite support and verifying the positivity constraint. Since our method follows the projection framework of generalized sampling, it offers the prospect of avoiding aliasing despite not using ideal pre-filters. We foresee additional developments, in particular, by using functions other than B-splines or implementing non-orthogonal projections.

\section{ACKNOWLEDGEMENT}

This research was funded by the Swiss National Science Foundation, Grants 200021_159227 "Computational Methods for Temporal Super-resolution Microscopy," 206021_164022 "Platform for Reproducible Acquisition, Processing, and Sharing of Dynamic, Multi-Modal Data," and "200020_179217, Computational biomicroscopy: advanced image processing methods to quantify live biological systems."

\section{REFERENCES}

[1] Vermot, J., Fraser, S. E., and Liebling, M., "Fast fluorescence microscopy for imaging the dynamics of embryonic development.," HFSP journal 2(3), 143-55 (2008).

[2] Bub, G., Tecza, M., Helmes, M., Lee, P., and Kohl, P., "Temporal pixel multiplexing for simultaneous high-speed, high-resolution imaging.," Nat. Methods 7(3), 209-11 (2010).

[3] Shechtman, E., Caspi, Y., and Irani, M., "Space-time super-resolution," IEEE Trans. Pattern Anal. Mach. Intell. 27(4), 531-545 (2005).

[4] Li, T., He, X., Teng, Q., Wang, Z., and Ren, C., "Space-time super-resolution with patch group cuts prior," Signal Process-Image 30, 147-165 (2015).

[5] Agrawal, A., Gupta, M., Veeraraghavan, A., and Narasimhan, S. G., "Optimal coded sampling for temporal super-resolution," in $[C V P R], 599-606$ (2010). 
[6] Pournaghi, R. and Wu, X., "Coded Acquisition of High Frame Rate Video," IEEE Trans. Image Process. 23(12), 5670-5682 (2013).

[7] Tsai, T.-H., Llull, P., Yuan, X., Carin, L., and Brady, D. J., "Spectral-temporal compressive imaging," Opt. Lett. 40(17), 4054-4057 (2015).

[8] Koller, R., Schmid, L., Matsuda, N., Niederberger, T., Spinoulas, L., Cossairt, O., Schuster, G., and Katsaggelos, A. K., "High spatio-temporal resolution video with compressed sensing," Opt. Express 23(12), $15992(2015)$.

[9] Llull, P., Liao, X., Yuan, X., Yang, J., Kittle, D., Carin, L., Sapiro, G., and Brady, D. J., "Coded aperture compressive temporal imaging," Opt. Express 21(9), 1289-1306 (2013).

[10] Chan, K. G., Streichan, S. J., Trinh, L. A., and Liebling, M., "Simultaneous temporal superresolution and denoising for cardiac fluorescence microscopy," IEEE Trans. Comput. Imaging 2(3), 348-358 (2016).

[11] Veeraraghavan, A., Reddy, D., and Raskar, R., "Coded strobing photography: Compressive sensing of high speed periodic videos," IEEE Transactions on Pattern Analysis and Machine Intelligence 33, 671-686 (Apr. 2011).

[12] Bosse, J. B., Tanneti, N. S., Hogue, I. B., and Enquist, L. W., "Open LED illuminator: A simple and inexpensive LED illuminator for fast multicolor particle tracking in neurons," PLOS ONE 10, 1-21 (Nov. 2015).

[13] Staudt, D. W., Liu, J., Thorn, K. S., Stuurman, N., Liebling, M., and Stainier, D. Y. R., "Highresolution imaging of cardiomyocyte behavior reveals two distinct steps in ventricular trabeculation," Development 141(3), 585-593 (2014).

[14] Raskar, R., Agrawal, A., and Tumblin, J., "Coded exposure photography: Motion deblurring using fluttered shutter," ACM Trans. Graph. 25, 795-804 (July 2006).

[15] Gorthi, S. S., Schaak, D., and Schonbrun, E., "Fluorescence imaging of flowing cells using a temporally coded excitation," Opt. Express 21, 5164-5170 (Feb. 2013).

[16] Unser, M., "Sampling - 50 years after Shannon," Proceedings of the IEEE 88(4), 569-587 (2000).

[17] Chacko, N., Liebling, M., and Blu, T., "Discretization of continuous convolution operators for accurate modeling of wave propagation in digital holography," J. Opt. Soc. Am. A 30, 2012-2020 (Oct. 2013).

[18] Gedalyahu, K., Tur, R., and Eldar, Y. C., "Multichannel sampling of pulse streams at the rate of innovation," IEEE Transactions on Signal Processing 59, 1491-1504 (Apr. 2011).

[19] Unser, M., Aldroubi, A., and Eden, M., "The L2 Polynomial Spline Pyramid," IEEE Transactions on Pattern Analysis and Machine Intelligence 15(4), 364-379 (1993).

[20] Jaques, C., Pignat, E., Calinon, S., and Liebling, M., "Temporal Super-Resolution Microscopy Using a Hue-Encoded Shutter," submitted .

[21] Pitrone, P. G., Schindelin, J., Stuyvenberg, L., Preibisch, S., Weber, M., Eliceiri, K. W., Huisken, J., and Tomancak, P., "OpenSPIM: an open-access light-sheet microscopy platform," Nat. Methods 10, 598-599 (June 2013). 